https://doi.org/10.21122/1029-7448-2020-63-3-197-211

УДК 621.31 .83 .52

\title{
Исследование переходных процессов электропривода с синхронным двигателем с постоянными магнитами при линейном изменении частоты питающего напряжения
}

\author{
Б. И. Фираго ${ }^{1)}$, С. В. Александровский ${ }^{1)}$ \\ ${ }^{1)}$ Белорусский национальный технический университет (Минск, Республика Беларусь) \\ (c) Белорусский национальный технический университет, 2020 \\ Belarusian National Technical University, 2020
}

Реферат. Синхронные двигатели с электромагнитным возбуждением и возбуждением от постоянных магнитов используются в различных промышленных установках, включая грузоподъемные машины и механизмы. Однако в большинстве случаев для регулирования скорости двигателей используется зависимое задание частоты питающего двигатель напряжения, т. е. векторное управление. В последнее время появился интерес к использованию частотнорегулируемых синхронных электроприводов с независимым заданием частоты питающего двигатель напряжения (скалярное частотное управление), так как они проще, чем электроприводы с векторным управлением. В то же время по сравнению с частотно-регулируемыми асинхронными электроприводами синхронные имеют меньшие потери мощности, жесткие механические характеристики без обратной связи по скорости, самый простой закон частотного управления - пропорциональный, который, однако, позволяет электродвигателю развивать почти максимальный момент в заданном частотном диапазоне благодаря постоянному магнитному потоку. В статье рассматривается аналитическое исследование переходных процессов электропривода с синхронным двигателем с постоянными магнитами (СДПМ) без демпферной обмотки при линейном изменении частоты питающего двигатель напряжения и линеаризации угловой характеристики. Получены аналитические выражения для угловой скорости и электромагнитного момента СДПМ при пуске, торможении, набросе и сбросе нагрузки. Расчеты угловой скорости и электромагнитного момента СДПМ по этим формулам позволяют оценить качество переходного процесса и определить максимальный электромагнитный момент двигателя, который не должен превышать допустимой величины. Показано, что при постоянном статическом моменте электропривод на основе СДПМ с обычным скалярным частотным управлением имеет незатухающие гармонические колебания скорости, что не позволяет применять его в установках с постоянным статическим моментом. Для устойчивой работы синхронного электропривода в установившемся режиме при постоянном статическом моменте предложен способ скалярного частотного управления электроприводом на основе СДПМ, где применяется отрицательная обратная связь по ускорению ротора. Результаты его расчета сопоставлены с результатами имитационного моделирования с использованием СДПМ фирмы OMRON типа SGMSH-50D мощностью 5 кВт и напряжением 400 В. Сравнение результатов показало эффективность применения предложенного способа управления по демпфированию колебаний СДПМ при постоянном статическом моменте.

Ключевые слова: синхронный двигатель с постоянными магнитами, частотное управление, обратная связь, переходные процессы

Для цитирования: Фираго, Б. И. Исследование переходных процессов электропривода с синхронным двигателем с постоянными магнитами при линейном изменении частоты питающего напряжения / Б. И. Фираго, С. В. Александровский // Энергетика. Изв. высси. учеб. заведений и энерг. объединений СНГ. 2020. Т. 63, № 3. C. 197-211. https://doi.org/10. 21122/1029-7448-2020-63-3-197-211

\begin{tabular}{ll}
\hline Адрес для переписки & Address for correspondence \\
Фираго Бронислав Иосифович & Firago Bronislav I. \\
Белорусский национальный технический университет & Belarusian National Technical University \\
просп. Независимости, 65/11, & $65 / 11$, Nezavisimosty Ave., \\
220013, г. Минск, Республика Беларусь & 220013 , Minsk, Republic of Belarus \\
Тел.: +375 17 293-95-61 & Tel.: +375 17 293-95-61 \\
eapu@bntu.by & eapu@bntu.by \\
\hline
\end{tabular}




\title{
Investigation of Transients of the Electrical Drive with a Permanent Magnet Synchronous Motor under a Ramp of Supply Voltage Frequency
}

\author{
B. I. Firago ${ }^{1)}$, S. V. Aleksandrovsky ${ }^{1)}$ \\ ${ }^{1)}$ Belarusian National Technical University (Minsk, Republic of Belarus)
}

\begin{abstract}
Electromagnetic excitation synchronous motors or permanent magnet excitation synchronous motors are used in various industrial facilities, including hoisting machines and mechanisms. However, in the most cases for the motor angular velocity control a dependent frequency reference, i.e. vector control, is used. Recently, there has been interest in using frequency-controlled synchronous electric drives with independent frequency setting of the motor supply voltage (i.e. with scalar frequency control), since they are simpler than electric drives with vector control. At the same time, in comparison with frequency-controlled asynchronous electric drives, synchronous drives lose less power, they are of rigid mechanical characteristics without speed feedback, and of the simplest law of frequency control, viz. of a proportional one, which, however, allows the electric motor to develop almost the maximum moment in a given frequency range due to constant magnetic flux. The article concerns an analytical study of the transients of a permanent magnet synchronous motor (PMSM) electric drive without a damping winding under a ramp of supply voltage and the linearization of the angular characteristic. Analytical expressions for the PMSM angular velocity and electromagnetic torque during starting, braking, loading up and loading down of the synchronous motor have been obtained. Calculations of the PMSM angular velocity and electromagnetic with the use of these formulae make it possible to estimate quality of the transients under examination and to determine an electromagnetic torque maximum value that must not exceed a specified one. It has been shown that under constant load torque the permanent magnet synchronous motor drive at usual scalar frequency control has the continuous angular velocity oscillations. To have a steady-state stable operation of PMSM electric drive under a constant load torque, the authors of the article have proposed a technique for the scalar frequency control of PMSM drive using a feed-back on the basis of synchronous motor rotor acceleration. The results of transients' calculations have been compared with the results of computer modeling of the PMSM electric drive on the basis of OMRON motor of the SGMSH-50D type (rated power is $5 \mathrm{~kW}$ and the rated voltage is $400 \mathrm{~V}$ ). The comparison of the results demonstrated the effectiveness of the proposed control method for damping PMSM vibrations at a constant static moment.
\end{abstract}

Keywords: permanent magnet synchronous motor, frequency control, feed-back, transients

For citation: Firago B. I., Aleksandrovsky S. V. (2020) Investigation of Transients of the Electrical Drive with a Permanent Magnet Synchronous Motor under a Ramp of Supply Voltage Frequency. Energetika. Proc. CIS Higher Educ. Inst. and Power Eng. Assoc. 63 (3), 197-211. https://doi.org/ 10.21122/1029-7448-2020-63-3-197-211 (in Russian)

\section{Введение}

В настоящее время наметился определенный интерес к синхронному электроприводу при скалярном частотном управлении [1-3]. Поэтому актуально рассмотреть переходные процессы синхронного электропривода при независимом задании частоты питающего напряжения. Для возможности аналитического исследования переходных процессов синхронного электропривода линеаризована угловая характеристика синхронного двигателя с постоянными магнитами (СДПМ) и принят постоянным статический момент нагрузки. Из разнообразия законов плавного изменения частоты напряжения, а следовательно, угловой синхронной скорости СДПМ 
в переходном процессе, остановимся на линейном, который наиболее распространен на практике. Рассматриваемые в статье вопросы базируются на нашей публикации [4].

В исследовании представлен аналитический метод расчета переходных процессов электропривода с синхронным двигателем с постоянными магнитами. Предложен способ управления СДПМ, при котором обеспечивается стабилизация угловой скорости ротора в установившемся режиме частотно-регулируемого синхронного двигателя без демпферной обмотки.

\section{Структурная схема и математическая модель линеаризованного синхронного электропривода}

Синхронные двигатели с постоянными магнитами при поверхностном расположении магнитов на роторе двигателя можно рассматривать как неявнополюсные синхронные двигатели (СД) с недовозбуждением [5]. СДПМ проектируются для электроприводов с векторным управлением, поэтому необходимости в установке на роторе демпферной (пусковой) короткозамкнутой обмотки нет. В этом случае электромагнитный момент двигателя в переходном процессе при скалярном частотном управлении будет состоять только из синхронного момента, определяемого по уравнению:

$$
M_{\text {син }}=\frac{M_{\text {ном }}}{\theta_{\text {ном }}} \theta=b \theta \text {, }
$$

где $b$ - магнитная жесткость СДПМ, обусловленная упругой связью ротора с вращающимся магнитным полем, $b=\frac{M_{\text {ном }}}{\theta_{\text {ном }}} ; M_{\text {ном }}-$ номинальный электромагнитный момент СДПМ; $\theta_{\text {ном }}-$ номинальный механический угол нагрузки СДПМ, $\theta_{\text {ном }}=\frac{\theta_{\text {эл.ном }}}{p_{\text {п }}} ; \theta_{\text {эл.ном }}-$ номинальный электрический угол нагрузки СДПМ; $p_{\text {п }}$ - число пар полюсов обмотки статора СДПМ; $\theta$ - угол нагрузки.

Угол рассогласования в положении магнитных осей статора и ротора, который еще называется углом нагрузки, определяется интегралом

$$
\theta=\int\left(\omega_{0}-\omega\right) d t
$$

где $\omega_{0}, \omega$ - текущее значение угловой скорости магнитного поля и ротора СДПМ.

Добавляем уравнение движения электропривода

$$
M-M_{\mathrm{c}}=J \frac{d \omega}{d t},
$$

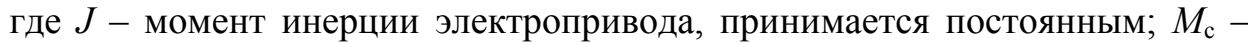
статический момент, также принимается неизменным. 
Уравнения (1) и (3) с учетом (2) запишем в операторной форме:

$$
\begin{aligned}
M(p) & =\frac{b}{p}\left[\omega_{0}(p)-\omega(p)\right] ; \\
p \omega(p) & =\frac{1}{J}\left[M(p)-M_{\mathrm{c}}(p)\right] .
\end{aligned}
$$

Этим двум уравнениям соответствует структурная схема, представленная на рис. 1.

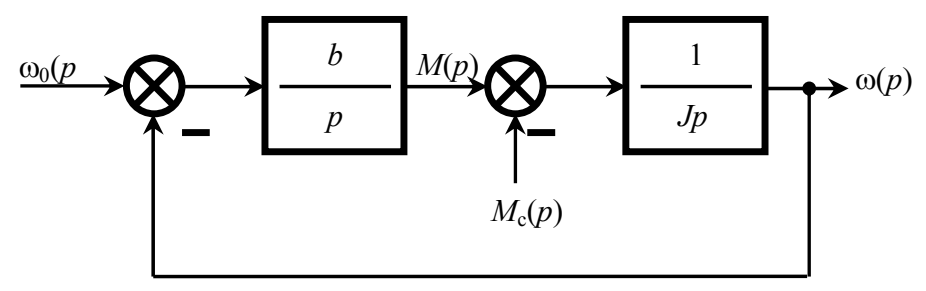

Puc. 1. Структурная схема линеаризованного синхронного электропривода

Fig. 1. A model block diagram of the linearized synchronous electrical drive

Исследуем переходный процесс синхронного электропривода при линейном изменении частоты $f_{1}$ питающего напряжения [6], а следовательно, и линейном изменении угловой скорости магнитного поля

$$
\omega_{0}=\varepsilon_{0} t+\omega_{0 \text { нач }}
$$

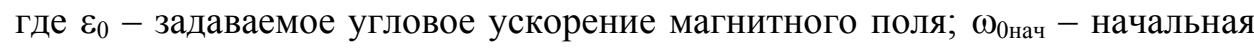
угловая скорость магнитного поля.

Математическая модель рассматриваемого синхронного электропривода при принятых допущениях представлена системой уравнений:

$$
\begin{gathered}
\omega_{0}=\varepsilon_{0} t+\omega_{0 \text { нач }} \\
M=b \int\left(\omega_{0}-\omega\right) d t \\
M-M_{\mathrm{c}}=J \frac{d \omega}{d t} .
\end{gathered}
$$

\section{Переходные процессы частотно-регулируемого электропривода с синхронным двигателем с постоянными магнитами без демпферной обмотки}

Статический момент нагрузки записывается уравнением [7]

$$
M_{\mathrm{c}}=C_{n} \omega^{n},
$$

где $\omega$ - текущее значение угловой скорости СДПМ; $C_{n}-$ коэффициент, определяемый для текущей угловой скорости как

$$
C_{n}=\frac{M_{\mathrm{c}}(\omega)}{\omega^{n}},
$$

$M_{\mathrm{c}}(\omega)$ - статический момент при данной угловой скорости $\omega ; n-$ показатель степени, который принимает значения $n=0,1,2$. 
На основании уравнения движения электропривода (3) получаем

$$
b \int\left(\omega_{0}-\omega\right) d t-C_{n} \omega^{n}=J \frac{d \omega}{d t} .
$$

Продифференцируем полученное уравнение

$$
b\left(\omega_{0}-\omega\right)-\frac{\partial M_{\mathrm{c}}}{\partial \omega} \frac{d \omega}{d t}=J \frac{d^{2} \omega}{d t^{2}} .
$$

Находим модуль жесткости характеристики статического момента при $\omega=\omega_{0}$ для данного показателя степени $n$, характеризующего статический момент:

$$
\left.\frac{\partial M_{\mathrm{c}}}{\partial \omega}\right|_{\omega=\omega_{0}}=\beta_{n}=C_{n} n \omega_{0}^{n-1} .
$$

В итоге получаем уравнение движения электропривода на основе СДПМ с обобщенным выражением статического момента

$$
b\left(\omega_{0}-\omega\right)-\beta_{n} \frac{d \omega}{d t}=J \frac{d^{2} \omega}{d t^{2}} .
$$

Разделим на коэффициент $b$ левую и правую части уравнения

$$
\omega_{0}-\omega-\frac{\beta_{n}}{b} \frac{d \omega}{d t}=\frac{J}{b} \frac{d^{2} \omega}{d t^{2}} .
$$

Как показано в [5], собственная частота электромеханических колебаний синхронного электропривода определяется выражением $\Omega_{0}=\sqrt{\frac{b}{J}}$, а отношение $\tau=\frac{\beta_{n}}{b}$ можно назвать электромагнитной постоянной времени синхронного электропривода.

В результате (7) преобразуется к виду

$$
\frac{1}{\Omega_{0}^{2}} \frac{d^{2} \omega}{d t^{2}}+\tau \frac{d \omega}{d t}+\omega=\omega_{0} .
$$

Данное дифференциальное уравнение имеет такое характеристическое выражение

$$
\frac{1}{\Omega_{0}^{2}} p^{2}+\tau p+1=0
$$

или

$$
p^{2}+\tau \Omega_{0}^{2} p+\Omega_{0}^{2}=0
$$

где $\tau \Omega_{0}^{2}=\frac{\beta_{n}}{b} \frac{b}{J}=\frac{\beta_{n}}{J}=\frac{1}{T_{m, n}} ; T_{m, n}-$ электромеханическая постоянная времени электропривода при данном виде статического момента. 
В итоге характеристическое уравнение принимает вид

$$
p^{2}+\frac{1}{T_{m, n}} p+\Omega_{0}^{2}=0
$$

Обозначим $\zeta_{n}=\frac{1}{2 T_{m, n}}-$ коэффициент затухания колебаний при данном виде статического момента.

Теперь характеристическое уравнение

$$
p^{2}+2 \zeta_{n} p+\Omega_{0}^{2}=0
$$

имеет корни $p_{1,2}=-\zeta_{n}+\sqrt{\zeta_{n}^{2}-\Omega_{0}^{2}}$.

Обычно $\zeta<<\Omega_{0}$, поэтому имеем комплексно-сопряженные корни $p_{1,2}=-\zeta_{n} \pm j \Omega_{\mathrm{p}}$, где $\Omega_{\mathrm{p}}-$ резонансная частота электромеханических колебаний синхронного электропривода, $\Omega_{\mathrm{p}}=\sqrt{\Omega_{0}^{2}-\zeta_{n}^{2}}$.

Как следует из приведенных выражений, коэффициент затухания и резонансная частота являются функцией характера статического момента, т. е. показателя степени $n$, который может принимать значения $n=0,1,2$.

Проанализируем корни характеристического уравнения, а следовательно, динамические свойства электропривода на основе СДПМ без демпферной обмотки со скалярным частотным управлением, когда синхронная угловая скорость ротора может находиться в диапазоне $\omega_{0 \min } \leq \omega_{0} \leq \omega_{0 \text { ном }}$.

При показателе степени $n=0$ имеем $M_{\mathrm{c}}=C_{n}=$ const, т. е. постоянный статический момент и, как видно из (6), нулевое значение модуля жесткости $\beta_{n}$ статического момента, приводящее к нулевому значению коэффициента затухания $\zeta_{n}$. В характеристическом уравнении (8) два мнимых корня $p_{1,2}= \pm j \Omega_{0}$, которые свидетельствуют о незатухающих электромеханических колебаниях синхронного электропривода с частотой $\Omega_{0}$ при отсутствии демпферной обмотки в СДПМ. Следовательно, СДПМ без демпферной обмотки нельзя использовать в скалярных системах частотного управления с постоянным статическим моментом.

На основании (4) можно получить дифференциальное уравнение для угловой скорости $\omega$ ротора синхронного двигателя

$$
\frac{J}{b} \frac{d^{2} \omega}{d t^{2}}+\omega=\omega_{0 \text { нач }}+\varepsilon_{0} t .
$$

Преобразуя (9), находим дифференциальное уравнение для электромагнитного момента СДПМ

$$
\frac{J}{b} \frac{d^{2} M}{d t^{2}}+M=M_{\text {с }}+M_{\text {дин }, 0}
$$


Для дифференциального уравнения угловой скорости ротора можно записать общее решение

$$
\omega=\omega_{0 \text { нач }}+\varepsilon_{0} t+\left(A e^{j \Omega_{0} t}+B e^{-j \Omega_{0} t}\right),
$$

где $A, B$ - постоянные интегрирования, определяемые из начальных условий:

$$
\begin{gathered}
\omega(t=0)=\omega_{\text {нач }} ; \\
\left.\frac{d \omega}{d t}\right|_{t=0}=\frac{M_{\text {нач }}-M_{\mathrm{c}}}{J}=\varepsilon_{\text {нач }} .
\end{gathered}
$$

Используем эти начальные условия для (11) и находим постоянные интегрирования $A$ и $B$, которые после подстановки в (11) и необходимых преобразований позволяют получить окончательное выражение для угловой скорости СДПМ в переходном процессе:

$$
\omega=\omega_{0 \text { нач }}+\varepsilon_{0} t-\Delta \omega_{\text {нач }} \cos \left(\Omega_{0} t\right)-\frac{\varepsilon_{0}-\varepsilon_{\text {нач }}}{\Omega_{0}} \sin \left(\Omega_{0} t\right),
$$

где $\Delta \omega_{\text {нач }}=\omega_{0 \text { нач }}-\omega_{\text {нач }}$.

Для дифференциального уравнения (10) начальные условия будут следующими:

$$
\begin{aligned}
& M(t=0)=M_{\text {нач }} ; \\
& \left.\frac{d M}{d t}\right|_{t=0}=b \Delta \omega_{\text {нач }} .
\end{aligned}
$$

Используя эти начальные условия совместно с (9) для нахождения решения уравнения (10), после преобразований получаем выражение для электромагнитного момента СДПМ в переходном процессе

$$
M=M_{\mathrm{c}}+M_{\text {дин }, 0}-\left[\left(M_{\text {нач }}-M_{\mathrm{c}}-M_{\text {дин }, 0}\right) \cos \left(\Omega_{0} t\right)+\frac{b \Delta \omega_{\text {нач }}}{\Omega_{0}} \sin \left(\Omega_{0} t\right)\right] .
$$

В качестве примера на рис. 2 приведены результаты расчета по полученным формулам (12) и (13) кривых переходного процесса - угловой скорости двигателя и электромагнитного момента электропривода с СДПМ типа SGMSH-50D фирмы OMRON мощностью 5 кВт и напряжением 400 B при коэффициенте инерции $K_{J}=2$, относительном статическом моменте $\mu_{\mathrm{c}}=0,4$ и времени линейного изменения угловой скорости магнитного поля $t_{0}=0,2 \mathrm{c}$. 
a

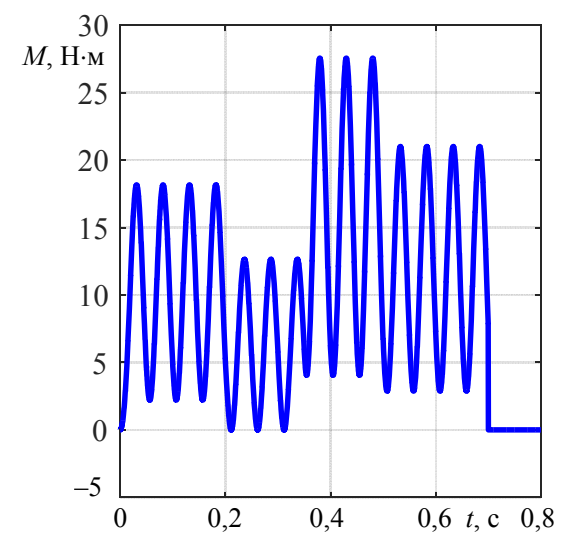

$\mathrm{b}$

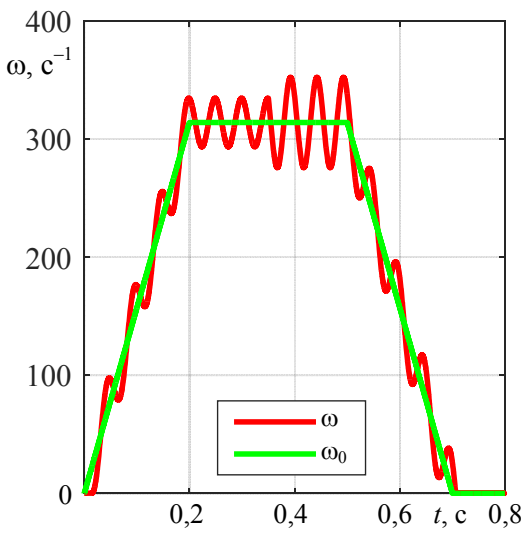

Puc. 2. Кривые переходных процессов при разгоне и торможении синхронного двигателя с постоянными магнитами типа SGMSH-50D при линейном изменении частоты питающего напряжения: a - электромагнитный момент электропривода; $\mathrm{b}$ - угловая скорость двигателя

Fig. 2. The curves of the transients of the SGMSH-50D type permanent magnet synchronous motor under accelerating and braking when the frequency of the supply voltage ramps: $a$ - electromagnetic torque of the electric drive; $b$ - motor angular velocity

Как видно из графиков, при отсутствии демпферной обмотки на роторе СДПМ переходные процессы электромагнитного момента и угловой скорости происходят в автоколебательном режиме. Внутренние демпфирующие свойства двигателя, обусловленные, например, механическими потерями, недостаточны для гашения колебаний, что делает неприемлемым применение СДПМ при скалярном частотном управлении без демпферной обмотки или специальных алгоритмов управления двигателем для механизмов с постоянным статическим моментом.

\section{Переходные процессы частотно-регулируемого \\ синхронного электропривода с постоянными магнитами \\ с отрицательной обратной связью по угловому ускорению}

В [8-10] при питании частотно-управляемых синхронных двигателей в качестве независимой переменной используется задаваемая угловая скорость $\omega_{3}$ в преобразователе частоты, а выходная частота $f_{1,3}$, или задаваемая угловая частота $\omega_{1,3}$, вычисляется из выражения

$$
\omega_{1,3}=\omega_{\text {эл, } 3}+\Delta \omega_{\text {эл }}=p_{\text {пा }}\left(\omega_{3}+\Delta \omega\right),
$$

где $\Delta \omega_{\text {эл }}$ определяется на основании модели СДПМ при вычислении активной мощности (так делают в векторных системах управления электродвигателем).

Назвать такой закон скалярным частотным управлением не совсем правомерно. В рассматриваемом случае применяется независимое задание частоты питающего СД напряжения и соответственно угловой частоты $\omega_{1,3}=p_{\text {пा }} \omega_{0,3}$, где $\omega_{0,3}-$ задаваемая синхронная угловая скорость СД. 
Угловая скорость ротора $\omega$ СД в переходном процессе определяется выражением

$$
\omega=\omega_{0,3}-\Delta \omega,
$$

где $\Delta \omega$ - изменение скорости относительно $\omega_{0,3}$ в переходном процессе.

При набросе нагрузки для СД с демпферной обмоткой, например, $\Delta \omega$ приведено в [5]. В других случаях, исходя из уравнений скорости в пере-

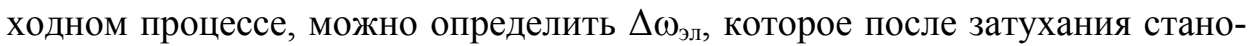
вится равным нулю и установившаяся угловая скорость $\omega_{\text {уст. }}=\omega_{0,3}=$ const.

Для обоснования возможности уменьшения колебаний ротора СД без демпферной обмотки при независимом задании частоты в скалярном частотном управлении СДПМ на рис. 3 представлена структурная схема электропривода при линеаризации угловой характеристики СДПМ и наличии отрицательной обратной связи по ускорению ротора [11].

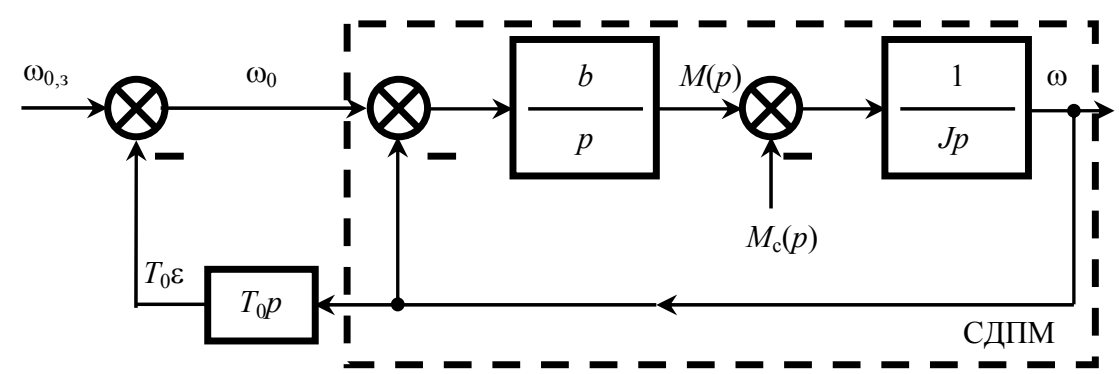

Рuc. 3. Структурная схема синхронного двигателя с постоянными магнитами при линеаризации угловой характеристики двигателя и наличии отрицательной обратной связи по угловому ускорению ротора

Fig. 3. A block diagram of the permanent magnet synchronous motor when the angular characteristic of the motor is linearized and there is a negative feedback on the angular acceleration of the rotor

При линейном задании имеем

$$
\omega_{0,3}=\omega_{0 \text { нач }}+\varepsilon_{0} t,
$$

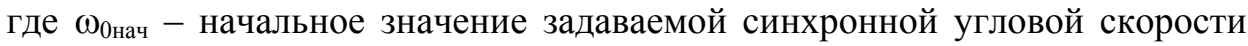
СДПМ в переходном процессе; $\varepsilon_{0}$ - задаваемое угловое ускорение ротора, $\varepsilon_{0}=\frac{\omega_{0 \text { кон }}-\omega_{0 \text { нач }}}{t_{0}} ; \omega_{0 \text { кон }}-$ конечное значение задаваемой синхронной угловой скорости СДПМ в переходном процессе; $t_{0}$ - время линейного изменения задаваемой синхронной угловой скорости.

На основании структурной схемы электропривода на рис. 3 определяем:

$$
\begin{gathered}
\Delta \omega_{0}=T_{0} \frac{d \omega}{d t} ; \\
\frac{d \omega}{d t}=\frac{M-M_{\mathrm{c}}}{J}=\frac{M_{\text {дин }}}{J} ;
\end{gathered}
$$




$$
\begin{gathered}
\omega_{0}=\omega_{0,3}-T_{0} \frac{M_{\text {дин }}}{J} ; \\
M=b \int\left(\omega_{0,3}-T_{0} \frac{M_{\text {дин }}}{J}-\omega\right) d t,
\end{gathered}
$$

где $b$ - магнитная жесткость СДПМ; $T_{0}$ - постоянная времени.

Продифференцируем последнее уравнение

$$
\frac{d M}{d t}=b\left[\omega_{0,3}-T_{0} \frac{M_{\text {дин }}}{J}-\omega\right] .
$$

Рассматривая переходный процесс в пределах линейного изменения $\omega_{0,3}$, можно получить

$$
M_{\text {дин }}=M_{\text {дин }, 0}=J \varepsilon_{0},
$$

что позволяет определить жесткость кривой динамического момента

$$
\beta=\frac{M_{\text {дин }}}{\omega_{0 \text { кон }}-\omega_{0 \text { нач }}}=\frac{J}{t_{0,3}} .
$$

Обозначим $\frac{J}{\beta}=t_{0,3}=T_{0}-$ постоянная времени, тогда $\frac{T_{0}}{J}=\frac{1}{\beta}$.

В результате

$$
\frac{d M}{d t}=b\left[\omega_{0,3}-\frac{M_{\text {дин }}}{\beta}-\omega\right]=\frac{b}{\beta}\left[\left(\omega_{0,3}-\omega\right) \beta-M_{\text {дин }}\right] .
$$

Отношение $\tau=\frac{\beta}{b}$ имеет размерность времени и в пределах принятых условий является постоянной величиной, поэтому называем $\tau$ постоянной времени.

Получаем дифференциальное уравнение

$$
\tau \frac{d M}{d t}+M=\beta\left(\omega_{0,3}-\omega\right)+M_{\mathrm{c}}
$$

Продифференцируем это выражение

$$
\tau \frac{d^{2} M}{d t^{2}}+\frac{d M}{d t}=\beta \varepsilon_{0}-\beta \frac{d \omega}{d t}=\beta \varepsilon_{0}-\beta \frac{M-M_{\mathrm{c}}}{J}=\beta \varepsilon_{0}-\left(M-M_{\mathrm{c}}\right) \frac{1}{T_{0}} .
$$

Имеем

$$
\tau T_{0} \frac{d^{2} M}{d t^{2}}+T_{0} \frac{d M}{d t}+M=\beta \varepsilon_{0} T_{0}+M_{\mathrm{c}}=J \varepsilon_{0}+M_{\mathrm{c}}=M_{\text {дин }}+M_{\mathrm{c}} .
$$

В окончательном виде получаем

$$
\tau T_{0} \frac{d^{2} M}{d t^{2}}+T_{0} \frac{d M}{d t}+M=M_{\text {дин }}+M_{\mathrm{c}} .
$$


Теперь найдем дифференциальное уравнение для угловой скорости ротора $\omega$ СД. Продифференцируем уравнение электромагнитного момента СДПМ с вычитанием $M_{\mathrm{c}}$ для данной структурной схемы:

$$
\begin{gathered}
b \int\left(\omega_{0, \text { нач }}+\varepsilon_{0} t-\omega-T_{0} \frac{M_{\text {дин }}}{J}\right) d t-M_{\mathrm{c}}=J \frac{d \omega}{d t} ; \\
b\left(\omega_{0 \text {,нач }}+\varepsilon_{0} t-\omega-T_{0} \frac{M_{\text {дин }}}{J}\right)=J \frac{d^{2} \omega}{d t^{2}} .
\end{gathered}
$$

Разделим на $b$ обе части уравнения

$$
\omega_{0, \text { нач }}+\varepsilon_{0} t-\omega-T_{0} \frac{M_{\text {дин }}}{J}=\frac{J}{b} \frac{d^{2} \omega}{d t^{2}} .
$$

Учитывая, что $\frac{J}{b}=\frac{J}{\beta} \frac{\beta}{b}=T_{0} \tau$, имеем

$$
T_{0} \tau \frac{d^{2} \omega}{d t^{2}}+T_{0} \frac{d \omega}{d t}+\omega=\omega_{0 \text { нач }}+\varepsilon t=\omega_{0,3} .
$$

Для обоснования принятой отрицательной обратной связи в системе управления «преобразователь частоты - синхронный двигатель с постоянными магнитами» в последнем дифференциальном уравнении пренебрегаем постоянной времени $\tau$ по сравнению с $T_{0}$. Тогда получаем для скорости ротора дифференциальное уравнение первого порядка

$$
T_{0} \frac{d \omega}{d t}+\omega=\omega_{0,3},
$$

которое запишем в виде

$$
T_{0} \frac{d \omega}{d t}=\omega_{0,3}-\omega .
$$

Воздействовать на изменение скорости можно только за счет изменения частоты. Как показано в [5], угловая скорость ротора СД в переходном процессе $\omega=\omega_{0,3}-\frac{d \theta}{d t}=\omega_{0,3}-\Delta \omega_{0}$.

Теперь получаем

$$
T_{0} \frac{d \omega}{d t}=\omega_{0,3}-\omega_{0,3}+\Delta \omega_{0}=\Delta \omega_{0} .
$$

Это выражение положено в основу отрицательной обратной связи для уменьшения колебаний скорости электропривода с СДПМ без демпферной обмотки при независимом задании частоты в скалярном частотном управлении синхронного двигателя.

Следует отметить, что $T_{0}$ является постоянной величиной только на интервале линейного изменения частоты (синхронной угловой скорости). 
При выходе на $\omega_{0,3}=\omega_{0 \text { кон }}=$ const значение $T_{0}=J / \beta$ уменьшается, так как при данном $J$ величина $\beta$ будет возрастать.

Выражения (14) и (15) имеют характеристическое уравнение

$$
\tau T_{0} p^{2}+T_{0} p+1=0
$$

или

$$
p^{2}+\frac{1}{\tau} p+\frac{1}{\tau T_{0}}=0
$$

Обозначим $\xi=\frac{1}{2 \tau}$ и назовем коэффициентом затухания; $m=\frac{T_{0}}{\tau}-$ отношение постоянных времени.

Запишем $T_{0}=m \tau$, тогда $\tau T_{0}=m \tau^{2}$ и характеристическое уравнение принимает вид

$$
p^{2}+2 \xi p+\frac{1}{m \tau^{2}}=0
$$

или

$$
p^{2}+\frac{1}{\tau} p+\frac{1}{m \tau^{2}}=0
$$

Решением (16) будет выражение

$$
p_{1,2}=-\frac{1}{2 \tau} \pm \sqrt{\left(\frac{1}{2 \tau}\right)^{2}-\frac{1}{m \tau^{2}}}=-\frac{1}{2 \tau} \pm \frac{1}{2 \tau} \sqrt{\frac{m-4}{m}} .
$$

Если $m<4$, получаем комплексно сопряженные корни $p_{1,2}=-\xi \pm j \Omega_{\mathrm{p}}$, где $\Omega_{\mathrm{p}}=\frac{1}{2 \tau} \sqrt{\frac{4-m}{m}}-$ резонансная частота колебаний ротора двигателя.

Определим условия, при которых имеем

$$
m=\frac{T_{0}}{\tau}<4, \text { или } T_{0}<4 \tau .
$$

Учтем, что $\tau=\frac{\beta}{b}=\frac{J}{T_{0}} \frac{1}{b}$, тогда $T_{0}<4 \frac{J}{T_{0}} \frac{1}{b}$, или $T_{0}^{2}<4 \frac{J}{b}$.

Принимаем во внимание, что

$$
\Omega_{0}=\sqrt{\frac{b}{J}}
$$

где $\Omega_{0}$ - собственная частота электромеханических колебаний синхронного электропривода.

Получаем неравенства:

$$
T_{0}^{2}<\frac{4}{\Omega_{0}^{2}} ; \quad\left(\Omega_{0} T_{0}\right)^{2}<4, \text { или } \Omega_{0} T_{0}<2 .
$$

Из этих условий для данного СДПМ при известном моменте инерции электропривода $J$ можем по (17) вычислить $\Omega_{0}$, а затем найти время линей- 
ного задания $\omega_{0,3}(t)$ в переходном процессе $t_{0,3}=T_{0}<\frac{2}{\Omega_{0}}$, а также соответственно задаваемое ускорение $\varepsilon_{0,3}=\frac{\omega_{0 \text { кон }}-\omega_{0 \text { нач }}}{t_{0,3}}$ и задаваемый динамический момент в переходном процессе $M_{\text {дин, } 0,3}=J \varepsilon_{0,3}$. Также можно использовать величину $t_{0,3}=T_{0}$ в цепи обратной связи по ускорению в предложенной структурной схеме указанного синхронного электропривода со скалярным частотным управлением.

Представляет интерес рассмотреть переходный процесс при $m=2$, т. е. когда $T_{0}=2 \tau$, или $\tau=\frac{T_{0}}{2}$ :

$$
\frac{T_{0}}{2}=\frac{1}{T_{0}} \frac{1}{\Omega_{0}^{2}}=>T_{0}^{2}=\frac{2}{\Omega_{0}^{2}} ;\left(\Omega_{0} T_{0}\right)^{2}=2 \Rightarrow \Omega_{0} T_{0}=\sqrt{2},
$$

$T_{0}<\frac{\sqrt{2}}{\Omega_{0}}$ удовлетворяет условию (16).

В данном случае будет оптимальный переходный процесс. Следовательно, если нет каких-либо ограничений, то можно принять

$$
t_{0,3}=T_{0, \text { опт }}=\frac{\sqrt{2}}{\Omega_{0}}=\frac{1,41}{\sqrt{\frac{b}{J}}} .
$$

Выполним компьютерное моделирование переходных процессов электропривода с СДПМ типа SGMSH-50D фирмы OMRON мощностью 5 кВт и напряжением 400 B при линейном изменении частоты питающего напряжения и наличии отрицательной обратной связи по угловому ускорению при коэффициенте инерции $K_{J}=2$, относительном статическом моменте $\mu_{\mathrm{c}}=0,4$ и времени линейного изменения угловой скорости магнитного поля $t_{0}=0,25 \mathrm{c}$ (рис. 4 ).

Можно отметить, что в переходном процессе скорости при наличии отрицательной обратной связи по ускорению по сравнению с переходным процессом скорости при наличии демпферной обмотки при $t>(6 \div 8) \tau$ имеет место отклонение угловой скорости ротора $\omega$ от угловой скорости магнитного поля $\omega_{0}$ на величину

$$
\Delta \omega=\omega_{0}-\omega=\varepsilon_{0} T_{0} .
$$

Такое изменение угловой скорости ротора СД аналогично изменению угловой скорости двигателя постоянного тока независимого возбуждения (ДПТ НВ) или асинхронного двигателя с короткозамкнутым ротором (АД КЗР) при линейном задании $\omega_{0}$. В то же время в установившемся режиме, когда $\varepsilon_{0}=0$, в отличие от ДПТ НВ и АД КЗР, отклонение угловой скорости СДПМ $\Delta \omega=\omega_{0}-\omega=0$. 

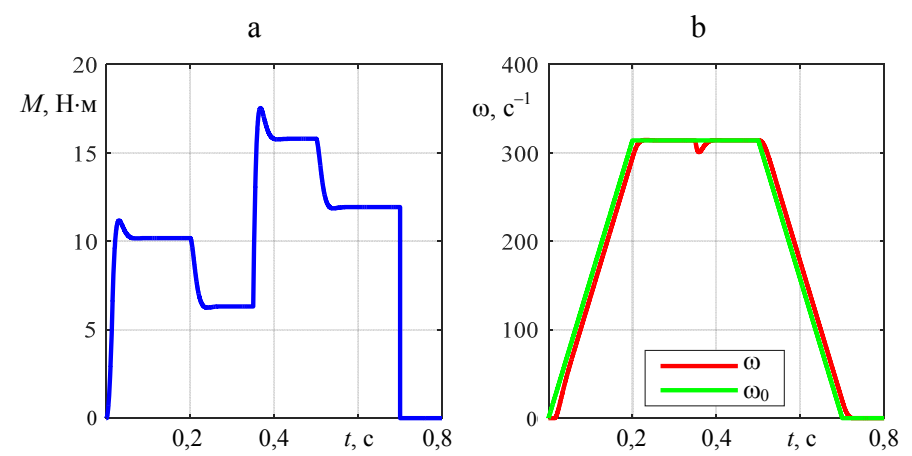

Puc. 4. Кривые переходных процессов при разгоне и торможении синхронного двигателя с постоянными магнитами типа SGMSH-50D при линейном изменении частоты питающего напряжения и наличии отрицательной обратной связи по угловому ускорению: $\mathrm{a}$ - электромагнитный момент; $\mathrm{b}$ - угловая скорость двигателя

Fig. 4. Accelerating and braking transients of the permanent magnet synchronous motor of the SGMSH-50D type under a ramp of frequency profile of supplied voltage and a negative feed-back on the angular acceleration: $a$ - electromagnetic torque; $b$ - motor angular velocity

\section{ВЫВОДЫ}

1. Разработана методика аналитического расчета переходных процессов частотно-регулируемого электропривода с синхронным двигателем с постоянными магнитами при линейном изменении частоты напряжения.

2. При скалярном частотном управлении, отсутствии демпферной обмотки в синхронном двигателе с постоянными магнитами и постоянном статическом моменте $(n=0)$ переходные процессы электромагнитного момента и угловой скорости ротора двигателя имеют незатухающие колебания, при которых невозможна нормальная работа машин и механизмов.

3. Предложен способ управления синхронным двигателем с постоянными магнитами, при котором обеспечивается стабилизация угловой скорости ротора в установившемся режиме частотно-регулируемого синхронного двигателя без демпферной обмотки. При этом используется независимое задание частоты в скалярном частотном управлении по пропорциональному закону $U_{1} / f_{1}=$ const, когда от сигнала заданной величины частоты питающего двигатель напряжения вычитается сигнал, пропорциональный первой производной угловой скорости ротора $\varepsilon$, с коэффициентом $T_{0}$, зависящим от момента инерции электропривода.

4. Разработанная методика расчета переходных процессов электропривода может быть применена на практике.

\section{ЛИТЕРАТУРА}

1. Мохсен, А. Перспективный электропривод мостовых кранов / А. Мохсен // Електромеханічні системи, методы моделювання та оптимізаціі: зб. матер. VI Міжнар. наук.-техн. конф. молод. учених і спец. у місті Кременчук, 8-10 квітня 2008 р. / Кремен. держ. політехн. ун-т; наук. ред. О. П. Чорний. Кременчук, 2008. С. 57-61.

2. Brock, S. Energy-Optimal V/F Control of Permanent Magnet Synchronous Motors for Fan Fpplications / S. Brock, T. Pajchrowski // Zeszyty Problemowe. Maszyny Elektryczne. 2011. No 92. P. 169-174.

3. Paitandi, S. Analysis, Design and Implementation of Sensorless V/F Control in a Surface-Mounted PMSM without Damper Winding / S. Paitandi, M. Sengupta // Sādhanā. 2017. Vol. 42, No 8. P. 1317-1333. https://doi.org/10.1007/s12046-017-0662-4.

4. Фираго, Б. И. Исследование переходных процессов в частотно-регулируемом синхронном электроприводе / Б. И. Фираго, С. В. Александровский // Энергетика. Изв. высш. 
учеб. заведений и энерг. объединений СНГ. 2016. Т. 59, № 6. С. 507-518. https://doi.org/ 10.21122/1029-7448-2016-59-6-507-518.

5. Фираго, Б. И. Регулируемые электроприводы переменного тока / Б. И. Фираго, Л. Б. Павлячик. Минск: Техноперспектива, 2006. 363 с.

6. Фираго, Б. И. Свойства, характеристики и параметры синхронного двигателя с постоянными магнитами при векторном и скалярном частотном управлении / Б. И. Фираго, С. В. Александровский // Энергетика. Изв. высш. учеб. заведений и энерг. объединений СНГ. 2019. Т. 62, № 3. С. 205-218. https://doi.org/10.21122/1029-7448-2019-62-3-205-218.

7. Фираго, Б. И. Теория электропривода / Б. И. Фираго, Л. Б. Павлячик. Минск: Техноперспектива, 2007. 585 с.

8. A Sensorless Stable V/F Control Method for Permanent-Magnet Synchronous Motor Drives / P. D. Perera Chandana [et al.] // IEEE Transactions on Industry Applications. 2003. Vol. 39, No 3. P. 783-791. https://doi.org/10.1109/tia.2003.810624.

9. Шмарин, Я. А. Повышение эффективности электропривода объемного гидронасоса многоколесной автотранспортной платформы / Я. А. Шмарин. Челябинск, 2017. 150 с.

10. Коршунов, А. Равноускоренный частотный пуск синхронного двигателя с постоянными магнитами на роторе [Электронный ресурс] / А. Коршунов // Силовая электроника. 2007. № 1. Режим доступа: https://power-e.ru/wp-content/uploads/2007 01 58.pdf.

11. Фираго, Б. И. Способ управления электроприводом переменного тока с синхронным двигателем с постоянными магнитами: заявка № a20190141 / Б. И. Фираго, С. В. Александровский. Опубл. 14.05.2019.

Поступила 27.12.2019 Подписана в печать 27.02.2020 Опубликована онлайн 29.05.2020

\section{REFERENCES}

1. Mokhsen A. (2008) Promising Electrical Drive for Overhead Cranes. Elektromekhanichni Sistemi, Metody Modelyuvannya ta Optimizatsii: Zb. Mater. VI Mizhnar. Nauk.-Tekhn. Konf. Molod. Uchenikh i Spets. u Misti Kremenchuk, 8-10 Kvitnya 2008 r. [Electromechanical Systems, Modelling and Optimization Methods: Proceedings of the VI Scientific and Technical Conference of Young Scientists and Professionals, Kremenchuk, 8-10 April 2018]. Kremenchuk, KPSU, 57-61 (in Russian).

2. Brock S., Pajchrowski T. (2011) Energy-Optimal V/F Control of Permanent Magnet Synchronous Motors for Fan Applications. Zeszyty Problemowe. Maszyny Elektryczne, (92), 169-174.

3. Paitandi S., Sengupta M. (2017) Analysis, Design and Implementation of Sensorless V/F Control in a Surface-Mounted PMSM without Damper Winding. Sädhanā, 42 (8), 1317-1333. https://doi.org/10.1007/s12046-017-0662-4.

4. Firago B. I., Aleksandrovsky S. V. (2016) The Study of Transient Processes of the FrequencyRegulated Synchronous Electric Drive. Energetika. Izvestiya Vysshikh Uchebnykh Zavedenii $i$ Energeticheskikh Ob'edinenii $S N G=$ Energetika. Proceedings of the CIS Higher Education Institutions and Power Engineering Associations, 59 (6), 507-518. https://doi.org/10.21122/ 1029-7448-2016-59-6-507-518 (in Russian).

5. Firago B. I., Pawlaczyk L. B. (2006) Regulated Alternative Current Electric Drives. Minsk, Tekhnoperspektiva Publ. 363 (in Russian).

6. Firago B. I., Aleksandrovsky S. V. (2019) Properties, Characteristics and Parameters of Permanent Magnet Synchronous Motors under Vector and Scalar Frequency Control. Energetika. Izvestiya Vysshikh Uchebnykh Zavedenii i Energeticheskikh Ob'edinenii SNG = Energetika. Proceedings of the CIS Higher Education Institutions and Power Engineering Associations, 62 (3), 205-218. https://doi.org/10.21122/1029-7448-2019-62-3-205-218 (in Russian).

7. Firago B. I., Pawlaczyk L. B. (2007) The Theory of Electrical Drives. Minsk, Tekhnoperspektiva Publ., 585 (in Russian).

8. Chandana Perera P. D., Blaabjerg F., Pedersen J. K., Thogersen P. (2003) A Sensorless, Stable $\mathrm{V} / \mathrm{F}$ Control Method for Permanent-Magnet Synchronous Motor Drives. IEEE Transactions on Industry Applications, 39 (3), 783-791. https://doi.org/10.1109/tia.2003.810624.

9. Shmarin Ya. A. (2017) Improving Performance of a Volumetric Hydraulic Pump Electrical Drive for Multi Wheel Platform. Chelyabinsk, 15 (in Russian).

10. Korshunov A. (2007) Equal Acceleration Frequency Starting of the Permanent Synchronous Motor with a Constant Acceleration. Silovaya Elektronika [Power Electronics], (1). Available at: https://power-e.ru/wp-content/uploads/2007_01_58.pdf (in Russian).

11. Firago B. I., Aleksandrovskii S. V. (2019) A Technique for Control of Alternating Current Electrical Drive with a Permanent Magnet Synchronous Motor. The Invertor's Application No a20190141 (in Russian).

Received: 27 December 2019 Accepted: 27 February 2020 Published online: 29 May 2020 